\title{
Mixed anxiety and depressive disorder outcomes: prospective cohort study in primary care
}

\author{
Kate Walters, Marta Buszewicz, Scott Weich and Michael King
}

\section{Background}

Mixed anxiety and depressive disorder (MADD) is common yet ill-defined, with little known about outcomes.

\section{Aims \\ To determine MADD outcomes over 1 year}

\section{Method}

We recruited 250 adults attending seven London general practices with mild-moderate distress. Three groups were defined using a diagnostic interview: MADD, other ICD-10 psychiatric diagnosis, no psychiatric diagnosis. We assessed symptoms of distress (General Health Questionnaire-28), quality of life (12-item Short Form Health Survey), general practitioner (GP) diagnosis and consultation rate at baseline, 3 months and 1 year.

\section{Results}

Two-thirds of participants with MADD had no significant psychological distress at 3 months (61\%) or 1 year (69\%). However, compared with those with no diagnosis, individuals had twice the risk of significant distress (incidence rate ratio 2.39, 95\% Cl 1.29-4.42) at 3 months but not 1 year, and persistently lower quality of life (mental health functioning). There was no significant difference in GP consultation rate/ diagnosis.

\section{Conclusions}

The majority with MADD improved, but individuals had an increased risk of significant distress at 3 months and a lower quality of life. As we cannot currently predict those with a poorer prognosis these patients should be actively monitored in primary care.

\section{Declaration of interest}

None.
Minor distress and emotional disorders are the commonest mental health problems encountered in primary care. ${ }^{1,2}$ Mixed anxiety and depressive symptoms that do not reach thresholds for formal psychiatric diagnosis make up nearly half of all psychological problems in the UK and are nearly four times more common than depressive disorder alone. ${ }^{3}$ Nevertheless, there has been much debate about the inclusion of mixed anxiety and depression as a new category in DSM-5, ${ }^{4}$ particularly since it is unclear when symptoms of milder distress that may be transient, understandable and part of normal human emotional responses should be seen as a disorder. There is little consensus about the impact of milder distress on mental health outcomes, quality of life, functional impairment and economic costs. Minor, 'subthreshold' or 'subclinical' disorders are commonly defined as having above threshold scores for significant distress on a validated measure, but without fulfilling the criteria for any other diagnosis according to the ICD- $10^{5}$ or the DSM-IV. ${ }^{6}$ In the proposed criteria for DSM $-5^{7}$ mixed anxiety and depression patients must have three to four symptoms of major depression, accompanied by anxious distress; whereas in ICD-10 mixed anxiety and depressive disorder (MADD) symptoms of anxiety and depression should be present (with no specified number) but neither should be clearly predominant or severe enough to justify an individual diagnosis, and some autonomic symptoms must be present. Since these criteria differ, studies cannot easily be compared across both systems. There are few studies reporting outcomes for minor distress with mixed anxiety and depression symptoms, in particular the group defined by ICD-10 as MADD. Those that exist are mostly small, methodologically heterogeneous and inconsistent in their findings. Some evidence has suggested that MADD may not be a stable diagnosis, with most either remitting or shifting to other diagnoses over time. ${ }^{8}$ A small number of studies have demonstrated an increased risk of major depression in those with either minor depression or 'subsyndromal' depressive symptoms ${ }^{9,10}$ as defined by DSM-IV. However, in absolute terms only a minority (around 10-20\%) appear to develop more severe disorders, with the majority improving over time. ${ }^{10-12}$ There is currently insufficient evidence to accurately estimate recovery rates for those with ICD-10 MADD. Our objectives in this study were to: describe the characteristics of people with MADD in primary care; determine their short- to medium-term outcomes in terms of persistent/recurrent psychological distress, associated quality of life, health services use and general practitioner (GP) diagnosis; and identify potential predictive factors for persistent/recurrent distress in people with MADD.

\section{Method}

This was a cohort study of consecutive people aged 18 years and over attending seven general practices in suburban and urban London, UK. Those unable to complete a written questionnaire in English, with significant cognitive impairment or incapacitating physical illness were excluded. Participants were selected in a two-stage sampling design from respondents to a waiting room screening questionnaire including the General Health Questionnaire 12-item version (GHQ-12). ${ }^{13}$ The methods/results of this initial screening are published in detail elsewhere. ${ }^{14}$ Participants who scored as having mild-moderate distress (in the range 2-6 on the GHQ-12) in the initial questionnaire survey were selected for a full psychiatric diagnostic interview using the Revised Clinical Interview Schedule (CIS-R). ${ }^{15,16}$ The CIS-R was used to categorise participants into three groups:

(a) MADD with significant symptoms and scoring above the CIS-R threshold (11/12) for a 'case' of psychological disorder but not reaching eligibility for any other formal ICD-10 diagnosis;

(b) other ICD-10 psychiatric diagnosis; and

(c) no psychiatric disorder. 
The computerised version of the CIS-R was chosen as a widely used and validated diagnostic instrument for common mental disorders in primary care and community settings. ${ }^{3,15,16}$ We used the ICD-10 definition of MADD as the DSM-IV/DSM-5 definition is currently still in proposed form.

\section{Measurements}

\section{Baseline}

All participants had a baseline interview including the CIS-R conducted either face to face or by telephone. ${ }^{17}$ They also completed several self-complete measures, including baseline psychological distress (GHQ-28) ${ }^{13,18,19}$ quality of life (version 2 of the 12-item Short Form Health Survey, SF-12), ${ }^{20,21}$ disability (World Health Organization Disability Assessment Schedule, WHO-DAS) ${ }^{22}$ somatic dysfunction (15-item somatic subscale of the Patient Health Questionnaire, PHQ) ${ }^{23}$ Information on sociodemographic characteristics (age, gender, ethnicity, employment, housing tenure), social factors (marital status, who they lived with, number of children, carer status), past psychiatric history, physical ill health (self-reported major physical illness, number of prescriptions categorised into $0-2$ or 3 or more), lifestyle (alcohol and drugs), life events in past year, treatments received for emotional problems (self-report and from GP records), help-seeking behaviour and help-seeking preferences were also collected.

\section{Outcomes}

Participants were followed up by postal questionnaire at 3 months and 1 year and case-note review of GP records at 1-year follow-up. The primary outcome was psychological distress, measured using the GHQ-28, a widely used and validated measure containing four subscales of anxiety, depression, somatic symptoms and social dysfunction. ${ }^{13,18,19}$ Secondary outcomes were quality of life (SF-12), GP diagnosis and GP consultation rate.

\section{Sample size}

We chose to sample people who scored in the low/moderate range at screening on the GHQ-12 in order to identify a sufficiently large sample of people with MADD to potentially identify predictors of outcome in this group. Using conservative estimates that $30 \%$ of our MADD group and $8 \%$ of our no diagnosis (control) group would be GHQ-28 'cases' at 1 year follow-up, ${ }^{24}$ at $90 \%$ power and 0.05 level of significance this would require 74 participants in each of these arms.

\section{Data analysis}

Analysis was performed using Stata release 10.0 software for Windows. Unadjusted associations between outcomes and main explanatory variables and each potential confounder were tested using two-tailed chi-squared, $t$ - and equality of medians tests. Colinearity was assessed for conceptually similar variables by cross-tabulation and scatter plots/correlation as appropriate. Given there was a marked right skewed distribution on the GHQ-28 at both 3 months and 1 year, with a large proportion of respondents scoring zero, it was necessary to dichotomise the outcome measure and choose an acceptable and valid case threshold to indicate 'caseness'. We calculated a receiver operating characteristics (ROC) curve against the baseline CIS-R scores for our sample. We chose a relatively high case threshold of $8 / 9$ for further analysis, as the threshold that achieved an adequate level of specificity $(79.6 \%)$, while maintaining a reasonable, albeit lower level of sensitivity $(68.6 \%)$.

Modified Poisson modelling was used for our main outcome the GHQ-28, using a dichotomous case/non-case definition of outcome, and for the secondary outcomes of GP diagnosis and GP consultation rate. Quality of life (SF-12) was sufficiently normally distributed to fit population-averaged panel-data models using generalised estimating equations (GEE). The variables from each group of potentially colinear variables that had the strongest association with outcomes and exposures on crude analysis and on theoretical grounds from the literature were selected for the modelling process. To avoid overloading models and potential colinearity a number of the variables were omitted. Interactions were assessed between the main explanatory variables and each of the other key variables in the model and also between age and gender. Robust standard errors were used to account for clustering by GP practice. We used a complete case analysis for responders to the two follow-up postal questionnaires. Where a single item was missing from our main outcome measure (GHQ-28) we imputed the missing data in Stata based on the scores of the other items in that subscale. We conducted a sensitivity analysis of worst and best case scenarios for the imputations to determine the potential impact on our findings.

\section{Results}

\section{Sample characteristics}

\section{Response rates and attrition}

In total 1383/1528 (91\%) of consecutive adult GP attendees from a range of GP surgery sessions spread throughout the week completed a waiting room screening questionnaire. Of these, $304 / 447$ (68\%) individuals who were eligible for inclusion opted to participate in the main cohort study. Interviews were completed for 250/304 (82\%) participants (Fig. 1). Overall 193/ $250(77 \%)$ returned postal follow-up questionnaires at 3 months and $172 / 250(69 \%)$ at 1 year. In total $215 / 250(86 \%)$ returned questionnaires at either 3 months or 1 year and 153/250 (61\%) returned questionnaires at both 3 months and 1 year. Case-note follow-up data were obtained for 238/250 (95\%) participants.

\section{Non-responders and missing data}

The cohort study participants were similar to non-responders at baseline in terms of gender and GHQ-12 screening score (mean

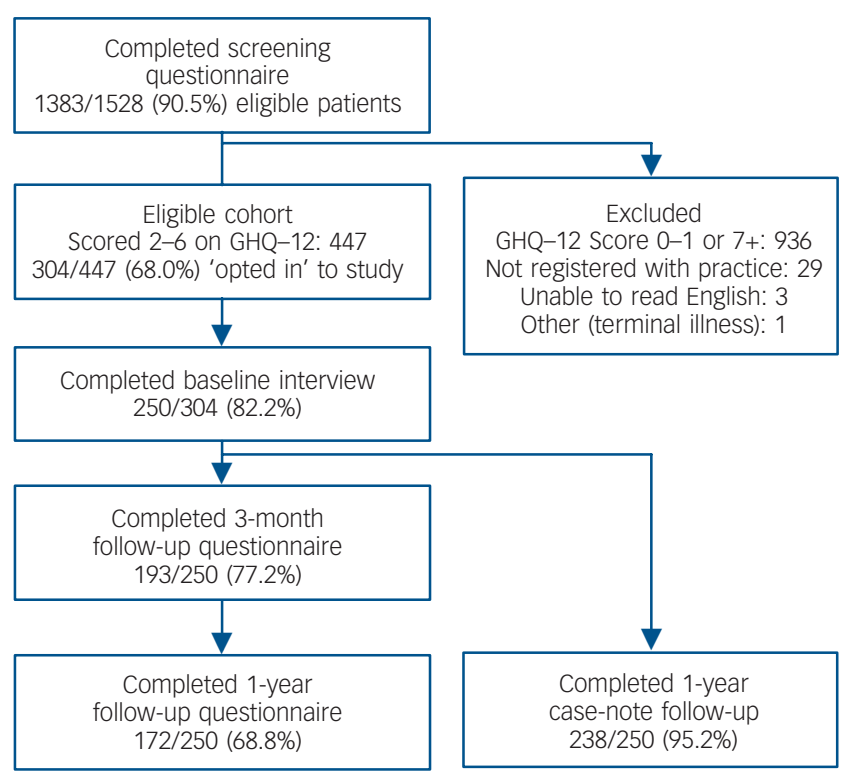

Fig. 1 Study response rates and attrition 
score 3.6 for both groups), but were slightly older (mean age $46 v$. 42 years for non-responders). Non-responders to the postal questionnaire at 3-month follow-up were slightly younger (mean age $41 v$. 48 years), from Black and minority ethnic groups ( $44 \% v$. $25 \%)$ and non-home owners (68\% v. 55\%). Results were similar for non-responders at 1 year. There was no difference in response rates for those in the MADD group compared with the no psychiatric diagnosis group, but more non-responders were in the other ICD-10 diagnosis group (21\% v. 11\%). Non-responders had slightly higher levels of psychological distress at baseline (GHQ-28 score 9.8, 95\% CI 8.0-11.6) than participants (GHQ-28 score 8.0, 95\% CI 7.3-8.8), but confidence intervals were overlapping. There were no meaningful differences in other sociodemographic characteristics. The sensitivity analysis of the imputation of single missing items of data for our main outcome, the GHQ-28, demonstrated no appreciable difference in our findings using best or worse case scenarios for the imputations.

\section{Sociodemographic characteristics}

The mean age for the whole sample was 46 years (95\% CI $44-48$ years) and $71 \%$ were women (online Table DS1). The sample was ethnically diverse, with only 137/250 (55\%) identifying themselves as 'White British' and a further 25/250 (10\%) as 'White European'. The largest Black and minority ethnic groups were 'Black African or Caribbean', and 'Asian' (Indian, Pakistani and Bangladeshi). The sample was socially diverse, with just under half $(120 / 250$, $48 \%)$ in paid employment and less than half (107/250, 43\%) were owner-occupiers. More than half reported a significant life event in the past year $(143 / 250,57 \%)$, the most common of which were major illness in themselves or someone close to them, changing or losing their job, relationship breakdown and bereavement.

\section{Baseline psychological symptoms, diagnoses and functioning}

The mean total scores were 8.4 (95\% CI 7.7-9.1) for the GHQ-28 and 13.5 (95\% CI 12.4-14.5) for the CIS-R within the sample as a whole at baseline (Table 1). Using the CIS-R 103/250 (41\%) had MADD, 34/250 (14\%) had another ICD-10 psychiatric diagnosis and $113 / 250(45 \%)$ had no diagnosis and scored lower than the threshold of 11 symptoms on the CIS-R. For those with other ICD-10 diagnoses the majority had mild, moderate or severe depressive disorder $(21 / 34,62 \%)$ and the remainder had panic disorder/agoraphobia (4/34), other specific phobias (6/34) or obsessive-compulsive disorder (2/34). The quality of life measured using the SF-12 in the overall sample was lower for the mental health subscale (mean 42.4, 95\% CI 40.9-43.8) than for the physical health subscale (mean 45.2, 95\% CI 43.7-46.7). Both of these scores were below general population norms. ${ }^{20}$ There was a wide variation in degree of disability, with a median score of 15 and an interquartile range (IQR) of 5-30 on the WHO-DAS 12-item screen.

\section{Univariable associations}

\section{Associations with MADD at baseline}

There were significant associations between some sociodemographic variables and MADD compared with those with no psychiatric diagnosis. These included female gender (odds ratio $(\mathrm{OR})=2.40$, 95\% CI 1.28-4.48), having children under 16 years of age $(\mathrm{OR}=1.83,95 \%$ CI $1.00-3.33)$, somatic dysfunction $(\mathrm{OR}=2.49,95 \%$ CI $1.28-4.84)$ and a self-reported past psychiatric history ( $\mathrm{OR}=2.56,95 \%$ CI 1.44-4.54) (online Table DS1). Those in the MADD group at baseline had increased psychological distress, including CIS-R overall score $(P<0.001)$, GHQ-28 overall score $(P<0.001)$ and all four subscales of the GHQ-28: anxiety $(P<0.001)$, depression $(\mathrm{P}=0.007)$, somatic symptoms $(P<0.001)$ and social dysfunction $(P<0.001)$ compared with those in the no psychiatric diagnosis group. They also had higher associated somatic dysfunction ('bothered a lot' by three or more somatic symptoms) using the PHQ somatic subscale $(P=0.003)$, disability WHO-DAS 12 -item screen $(P<0.001)$, number of disability days $(P<0.001)$ and lower quality of life on the mental health subscale of the SF-12 $(P<0.001)$ (Table 1$)$. There was no significant association with age or the physical health subscale of the SF-12.

\section{Primary outcome}

The median GHQ-28 score was significantly higher in both the MADD and other ICD-10 diagnosis groups when compared with the no psychiatric diagnosis group at all three time points (Table 2). The median GHQ-28 scores improved substantially in the MADD group at 3 months and 1 year, but remained higher in

\begin{tabular}{|c|c|c|c|c|c|}
\hline Variable & $\begin{array}{l}\text { Overall } \\
(n=250)\end{array}$ & $\begin{array}{l}\text { MADD } \\
\text { group } \\
(n=103)\end{array}$ & $\begin{array}{c}\text { Other ICD-10 } \\
\text { diagnosis group } \\
(n=34)\end{array}$ & $\begin{array}{l}\text { No diagnosis } \\
\text { group } \\
(n=113)\end{array}$ & $\begin{array}{l}\text { MADD } v . \text { no } \\
\text { diagnosis } \\
\text { group, } P\end{array}$ \\
\hline CIS-R: total score, mean $(95 \% \mathrm{Cl})(n=250)$ & $13.5(12.4-14.5)$ & $17.9(16.9-18.9)$ & $24.1(21.3-26.8)$ & $6.2(5.6-6.8)$ & $0.001^{a}$ \\
\hline \multicolumn{6}{|l|}{ GHQ-28 $(n=246)$} \\
\hline $\begin{array}{l}\text { Overall, mean score (95\% Cl) } \\
\text { Subscales, median (IQR) }\end{array}$ & $8.4(7.7-9.1)$ & 10.3(9.4-11.2) & $12.6(10.5-14.8)$ & $5.4(4.6-6.2)$ & $0.001^{a}$ \\
\hline Anxiety & $3(1-5)$ & $4(2-5)$ & $4(2-6)$ & $1(0-3)$ & $<0.001^{b}$ \\
\hline Somatic & $3(1-4)$ & $4(2-5)$ & $4.5(2-6)$ & $2(1-3)$ & $<0.001^{\mathrm{b}}$ \\
\hline Social dysfunction & $1(0-3)$ & $2(1-4)$ & $2(1-5)$ & $1(0-2)$ & $<0.001^{\mathrm{b}}$ \\
\hline Depression & $0(0-0)$ & $0(0-1)$ & $1(0-2)$ & $0(0-0)$ & $0.007^{b}$ \\
\hline \multicolumn{6}{|l|}{ Quality of life (SF-12 score), mean (95\% Cl) $(n=250)$} \\
\hline Physical health functioning & $45.2(43.7-46.7)$ & $44.9(42.5-47.4)$ & $41.0(36.1-45.9)$ & $46.7(44.7-48.7)$ & $0.27^{\mathrm{a}}$ \\
\hline Mental health functioning & $42.4(40.9-43.8)$ & $38.3(36.3-40.3)$ & $35.0(31.8-38.2)$ & $48.1(46.3-50.0)$ & $<0.001^{a}$ \\
\hline \multirow{2}{*}{$\begin{array}{l}\text { Somatic dysfunction (PHQ-15 score), median (IQR) } \\
\text { Disability (WHO-DAS score), median (IQR) }(n=241)\end{array}$} & $1(0-3)$ & $1(0-3)$ & $3(1-4)$ & $1(0-2)$ & $0.003^{b}$ \\
\hline & $15(5-30)$ & $19(10-30)$ & $26(14.5-30)$ & $10(2-20)$ & $<0.001^{\mathrm{b}}$ \\
\hline Disability days, ${ }^{\mathrm{C}}$ median (IQR) & $5(0-14)$ & $6.5(2-15)$ & $15(4.5-30)$ & $2(0-8)$ & $0.001^{b}$ \\
\hline \multicolumn{6}{|c|}{$\begin{array}{l}\text { CIS-R, Revised Clinical Interview Schedule; GHQ-28, 28-item General Health Questionnaire; MADD, mixed anxiety and depressive disorder; PHQ-15, } 15 \text {-item Patient Health } \\
\text { Questionnaire; SF-12, } 12 \text {-item Short Form Health Survey; IQR, interquartile range; WHO-DAS, World Health Organization - Disability Assessment Schedule. } \\
\text { a. From unpaired } t \text {-test with equal variances comparing MADD with the no diagnosis group. Significant results are in bold. } \\
\text { b. From equality of medians test comparing MADD with the no diagnosis group. Significant results are in bold. } \\
\text { c. Number of days in past } 30 \text { when activities were cut down or stopped because of disability. }\end{array}$} \\
\hline
\end{tabular}




\begin{tabular}{|c|c|c|c|c|c|c|}
\hline \multirow[b]{3}{*}{ Group } & \multicolumn{6}{|c|}{ GHQ-28 score } \\
\hline & \multicolumn{2}{|c|}{ Baseline } & \multicolumn{2}{|c|}{3 months } & \multicolumn{2}{|c|}{1 year } \\
\hline & Median (IQR) & $P^{\mathrm{a}}$ & Median (IQR) & $P^{a}$ & Median (IQR) & $P^{a}$ \\
\hline No diagnosis & $4(2-8)$ & & $1(0-4)$ & & $1(0-4)$ & \\
\hline Mixed anxiety and depressive disorder & $10(7-13)$ & $<0.001$ & $5(2-10)$ & $<0.001$ & $4.5(0-10)$ & 0.003 \\
\hline Other ICD-10 disorder & $13(9-15)$ & $<0.001$ & $9.5(2-13)$ & 0.022 & $14(4-19)$ & $<0.001$ \\
\hline
\end{tabular}

the other ICD-10 diagnosis group. In the overall sample the only significant associations of sociodemographic variables with being a GHQ-28 'case' for psychological distress (using the 8/9 threshold) were with self-reported past psychiatric history at both 3 months (crude OR $=2.03,95 \%$ CI 1.04-3.96) and 1 year (crude $\mathrm{OR}=4.05,95 \% \mathrm{CI} 1.84-8.90)$ and with somatic dysfunction at both 3 months (crude OR $=3.15,95 \%$ CI 1.49-6.69) and 1 year (crude $\mathrm{OR}=2.74,96 \% \mathrm{CI} 2.74-6.10$ ). In the MADD group $47 /$ $77(61 \%)$ were below the $8 / 9$ threshold for psychological distress at 3 months and $48 / 70(69 \%)$ at 1 year follow-up.

\section{Multivariable analysis}

\section{Psychological distress}

Those in the MADD group were more than twice as likely as the no psychiatric diagnosis group to be a GHQ-28 case at both 3 months and 1 year (Table 3 ). When this was adjusted for age, gender, past psychiatric history, somatic dysfunction and clustering by practice the risk was reduced, but remained significant at 3 months, with an adjusted incidence rate ratio (IRR) of 2.39 (95\% CI 1.29-4.42). By 1-year follow-up, the point estimates for being a GHQ-28 case were still raised in the MADD group, but the disparity with the no psychiatric diagnosis group was no longer significant (IRR $=1.73,95 \%$ CI 0.87-3.47). For the other ICD-10 disorders group the risk of being a GHQ case of distress at follow-up compared with risk in the no psychiatric diagnosis group was increased still further, with a nearly fourfold increase in risk at 3 months and a nearly fivefold increased risk at 1 year. After adjustment for confounding factors the point estimates were reduced slightly, but remained significant at both 3 months (IRR $=2.87,95 \%$ CI $1.41-5.88)$ and 1 year $(\mathrm{IRR}=3.18,95 \%$ CI $1.56-6.51)$.

\section{Quality of life}

There was a significantly lower quality of life (mental functioning) for both the MADD $(-5.36,95 \%$ CI -8.40 to -2.31$)$ and other
ICD-10 diagnosis $(-4.92,95 \%$ CI -8.80 to -1.64$)$ groups in comparison to the no diagnosis group at 3 months, and this was of a similar degree for both groups (Table 4). At 1-year follow-up the lowered mental functioning quality of life persisted at a similar level in the MADD group $(-5.04,95 \% \mathrm{CI}-8.43$ to $-1.64)$, but had markedly deteriorated in the other ICD-10 diagnoses group $(-12.09,95 \%$ CI -17.07 to -7.11$)$. There was no significant difference in physical functioning quality of life at either 3 months or 1 year for either the MADD or other ICD-10 diagnostic groups.

\section{Consultation rate}

There was no significant difference in GP consultation rates between those in the MADD group and the no diagnosis group (Table 4). The consultation rate was, however, significantly higher in the other ICD-10 diagnoses group, even after adjustment for comorbid physical illness and other demographic factors $(\mathrm{IRR}=1.46,95 \%$ CI 1.08-1.99).

\section{GP diagnosis}

There was no significant difference in a GP-reported diagnosis of a mental health problem between the MADD and no diagnosis groups over the year's follow-up (Table 4). Those with other ICD-10 disorders were, however, two and a half times more likely to have a mental health problem recorded in their case notes over the whole year follow-up period than those in the no diagnosis group $(\mathrm{IRR}=2.55,95 \%$ CI $1.36-4.75)$.

\section{Discussion}

\section{Main findings}

People with MADD were more likely to be women, have multiple somatic symptoms at baseline and a history of mental health difficulties when compared with those with no psychiatric

Table 3 Multivariable analysis: crude and adjusted risk of General Health Questionnaire (GHQ-28) caseness at 3 months and 1 -year follow-up in the mixed anxiety and depressive disorder (MADD) and other ICD-10 diagnoses groups ${ }^{\mathrm{a}}$

\begin{tabular}{|c|c|c|c|c|c|c|}
\hline \multirow[b]{2}{*}{ Model } & \multicolumn{3}{|c|}{ 3-month follow-up $(n=179)$} & \multicolumn{3}{|c|}{ 1-year follow-up ( $n=160$ ) } \\
\hline & $\begin{array}{l}\text { GHQ-28 } \\
\text { case, } n(\%)\end{array}$ & $\begin{array}{l}\text { Crude IRR } \\
(95 \% \mathrm{Cl})^{\mathrm{b}}\end{array}$ & $\begin{array}{l}\text { Adjusted }^{\mathrm{c}} \\
\text { IRR }(95 \% \mathrm{CI})^{\mathrm{b}}\end{array}$ & $\begin{array}{l}\text { GHQ-28 } \\
\text { case, } n(\%)\end{array}$ & $\begin{array}{l}\text { Crude IRR } \\
(95 \% \mathrm{Cl})^{\mathrm{b}}\end{array}$ & $\begin{array}{l}\text { Adjusted }{ }^{\mathrm{c}} \text { IRR, } \\
\qquad(95 \% \mathrm{Cl})^{\mathrm{b}}\end{array}$ \\
\hline No diagnosis ( 3 month $n=84,1$ year $n=72$ ) & $12(14.3)$ & 1.0 & 1.0 & $10(13.9)$ & 1.0 & 1.0 \\
\hline $\begin{array}{l}\text { Mixed anxiety depressive disorder } \\
(3 \text { month } n=77,1 \text { year } n=70)\end{array}$ & $30(39.0)$ & $2.73(1.50-4.95)$ & $2.39(1.29-4.42)$ & $22(31.4)$ & $2.26(1.15-4.44)$ & $1.73(0.87-3.47)$ \\
\hline $\begin{array}{l}\text { Other } I C D-10 \text { diagnosis ( } 3 \text { month } n=18 \text {, } \\
1 \text { year } n=18 \text { ) }\end{array}$ & $10(55.6)$ & $3.89(1.99-7.59)$ & $2.87(1.41-5.88)$ & $12(66.7)$ & $4.80(2.47-9.32)$ & $3.18(1.56-6.51)$ \\
\hline \multicolumn{7}{|c|}{$\begin{array}{l}\text { IRR, incidence rate ratio. } \\
\text { a. Results in bold are significant. } \\
\text { b. Incidence rate ratios for MADD and other ICD-10 disorders groups in comparison with the no psychiatric diagnosis group using maximum likelihood ratios using robust standard } \\
\text { errors. } \\
\text { c. Adjusted for age, gender, past psychiatric history, somatic dysfunction and clustering by practice using robust standard errors. }\end{array}$} \\
\hline
\end{tabular}


Table 4 Secondary outcomes: quality of life, general practitioner (GP) consultation rate and GP diagnosis over 1-year follow-up in mixed anxiety and depressive disorder and other ICD-10 disorders compared with the no diagnosis group ${ }^{a}$

3-month follow-up

1-year follow-up

12-item Short Form, difference in comparison with no diagnosis, ${ }^{b}$ mean $(95 \% \mathrm{Cl}$ )

Mental functioning

Mixed anxiety and depressive disorder

$-5.36(-8.40$ to -2.31$) \quad-5.04(-8.43$ to -1.64$)$

Other ICD-10 disorders

Physical functioning

Mixed anxiety and depressive disorder

$-4.92(-8.80$ to -1.64$) \quad-12.09(-17.07$ to -7.11$)$

Other ICD-10 disorders

$-1.66(-4.67$ to 1.34$)$

$-1.48(-4.59$ to 1.62$)$

$-3.58(-8.07$ to 0.92$)$

$-3.40(-7.98$ to 1.19$)$

GP consultations, IRR $(95 \% \mathrm{Cl})^{\mathrm{C}}$

Mixed anxiety and depressive disorder

$0.98(0.79$ to 1.20$)$

Other ICD-10 disorders

1.46 (1.08 to 1.99$)$

GP diagnosis, IRR $(95 \% \mathrm{Cl})^{\mathrm{C}}$

Mixed anxiety and depressive disorder

$1.27(0.72$ to 2.31$)$

Other ICD-10 disorders

$2.55(1.36$ to 4.75$)$

IRR, incidence rate ratio

a. Results in bold are significant

b. Difference in comparison with no psychiatric diagnosis group, adjusted for age, gender, past psychiatric history, somatic dysfunction and for clustering by practice using robust

standard errors.

c. Incidence rate ratios in comparison with the no psychiatric diagnosis group, adjusted for age, gender, past psychiatric history, somatic dysfunction, number of prescription

medications and major physical illness and for clustering by GP practice using robust standard errors.

d. Incidence rate ratios in comparison with no psychiatric diagnosis group, adjusted for age, gender, past psychiatric history and somatic dysfunction and for clustering by GP

practice using robust standard errors.

diagnosis. Around two-thirds of those with MADD did not have levels of distress high enough to reach the threshold to be considered distressed on the GHQ-28 at 3 months (61\%) or 1 year (69\%). However, those with MADD were more than twice as likely to be psychologically distressed 3 months from baseline as those with no diagnosis. Those with other ICD-10 diagnoses remained the most affected of the three groups at both 3 and 12 months. No variables were found to be significant predictors of persistent or recurrent distress in the MADD group at either 3 months or 1 year. The pattern of findings for the quality of life mental health functioning subscale was similar to those for psychological distress across the three groups, but there were no significant differences in physical health functioning. Participants with MADD attended their GP practice no more often than those without a diagnosis, and were no more likely to have a mental health problem recorded by their GP during the year's follow-up. Participants with other ICD-10 diagnoses were the most frequent users of GP services and were more likely to have a GP-recorded mental health problem.

\section{Strengths and limitations}

This is one of very few prospective studies specifically designed to measure the outcomes of people attending their general practice with MADD. The sample was recruited from those attending seven UK GP practices in urban/suburban settings, with excellent response rates to the initial screening questionnaire. There were lower rates of participation in the main cohort, but participants were similar in terms of age, gender and mean level of distress experienced when compared with those declining to take part. There was good follow-up of participants, with most (86\%) completing at least one of the two follow-up questionnaires and nearly complete $(95 \%)$ case-notes follow-up. Non-responders to follow-up questionnaires had slightly higher baseline levels of distress, although confidence intervals were overlapping. Attrition rates were similar for the MADD and no diagnosis groups, but slightly higher in the other ICD-10 diagnosis group, and the findings from this study may over-represent those with less severe symptoms.

All participants had between two and six symptoms of psychological distress on the baseline GHQ-12 screening questionnaire 2 weeks earlier. This means that the sample underrepresented both those with no symptoms of distress and those with severe distress, which would have the effect of biasing our study towards finding no difference between the groups. As the distribution of GHQ-28 scores at follow-up was so skewed towards complete recovery we were not able to analyse these as a continuous measure in our multivariable analysis. A higher case threshold was chosen for the GHQ-28 than is commonly used in other studies. ${ }^{13}$ This high threshold was chosen as it represented the best balance of sensitivity and specificity against the CIS-R within the sample. There was insufficient power to fully determine potential predictors of persistent distress/recovery within the MADD group. In common with any observational study there may have been unmeasured confounding.

\section{Interpretation in relation to existing literature}

The lack of statistically significant associations with sociodemographic factors in our study is consistent with the little existing work in the area, which has also shown a lack of strong associations with MADD. ${ }^{25-27}$ Those with MADD had higher disability and lower quality of life than the no diagnosis group at baseline, consistent with the literature..$^{27,28}$ Using the $8 / 9$ threshold on the GHQ-28, around two-thirds of those in the MADD group were non-cases at 3 months $(61 \%)$ and 1 year $(69 \%)$. These rates are broadly similar to the majority of studies evaluating MADD $25,26,29$ and somewhat higher than the WHO Collaborative study using a different instrument for case definition where only $49 \%$ recovered. $^{8}$ Recovery rates for MADD are lower than for adjustment disorder, a disorder with similar symptoms but differences in the nature of stressors, where studies suggest most symptoms resolve rapidly. ${ }^{30,31}$ The findings for MADD also contrast with the literature on comorbid threshold disorders where comorbid depression and anxiety or 'cothymia' has a worse prognosis with more associated disability and more persistent symptoms than either depression or anxiety disorders alone. ${ }^{32,33}$

It appears that, for the majority of those with MADD this can be considered a self-limiting condition, with improvements expected for most in the short and longer term. However, onethird of those with MADD had significant psychological distress 
at follow-up and those with MADD were more than twice as likely to be significantly distressed at 3 months follow-up when compared with the no diagnosis group, although this lessened to non-significant levels by 1 year. There was no other study in the literature of reasonable quality that reported the relative risk of psychiatric caseness at follow-up in comparison with a no psychiatric diagnosis group for those with MADD, with which we could directly compare our figures. Past studies on MADD have reported simple proportions who have either recovered or who are ICD-/DSM-defined psychiatric cases at followup. ${ }^{8,25,26,29}$ A number of studies have reported the risk of major depression in those with either minor depression or subsyndromal depression. These found increased odds of around fivefold of developing major depression for those with minor depression defined by DSM-IV or ICD-10 ${ }^{10,34}$ and fourfold for those with subsyndromal depressive symptoms. ${ }^{9,10}$ These studies were heterogeneous, using a variety of case definitions and outcome measures.

In this study, those with MADD had poorer mental health related quality of life than those with no psychiatric diagnosis, but a better quality of life than those with other ICD-10 diagnoses. This is consistent with previous published research. ${ }^{27,28,35}$ There was no evidence from this study that those with MADD consumed more health resources than those with no psychiatric diagnosis, since they did not consult their GP more frequently and did not receive more diagnoses of mental health problems. These findings contrast with past work in other countries, which has found higher health services use in those with minor distress and subthreshold disorders in comparison with those with no psychiatric symptoms. ${ }^{35}$ In our initial survey of 1357 people attending their GP less than one-half of those with mild-moderate distress reported wanting help if they were 'feeling stressed, worried or low and it was affecting their daily life. ${ }^{14}$

In our study no factors included in the models were identified as significant predictors of persistent distress within the MADD group at follow-up. There has been limited past research on this and no robust evidence for any one factor in predicting recovery. It is therefore currently difficult to predict which people with MADD have a worse or better prognosis, and further research is needed.

\section{Implications}

Our results suggest that for many people with MADD their symptoms will improve over time and around two-thirds will not be significantly distressed 3 months or 1 year later. However, those with MADD are more than twice as likely as those with no psychiatric diagnosis to have significant distress at 3 months, and also have persistently lower mental health related quality of life. This supports a recommendation of 'active monitoring' for those with MADD (discussion of problem/concerns, psychoeducation and active follow-up within 2 weeks), which is the current advice given to GPs for those with mild or subthreshold depressive symptoms. $^{36}$

Although those with MADD were at higher risk of psychological distress at follow-up, unlike those with other ICD-10 diagnoses, they did not present to their GP more frequently or have more diagnoses of mental health problems than the no diagnosis group. This situation could conceivably change if a policy of 'active monitoring' were followed in this group. We identified no strong predictors in our cohort to assist with identifying those at higher risk of persistent symptoms, but this was limited by our sample size. The MADD group appears heterogeneous, and it is currently unclear which subgroups are likely to have a poorer prognosis and reduced quality of life.
Further research is needed to address this. Including MADD as a diagnostic category in DSM-5 could lead to the medicalisation of many people who have minor, self-limiting symptoms of distress.

To conclude, many of those with MADD have a self-limiting course, but they are at short-term increased risk of significant distress, have a persistently lower quality of life in terms of mental health functioning and around one-third have significant symptoms of psychological distress at follow-up. Despite this, they are no more likely to seek help from their GP or be diagnosed with mental health problems over the following year than those with no psychiatric diagnosis. We cannot currently predict which people in this heterogeneous group have a better or worse prognosis.

Kate Walters, MRCGP, PhD, Marta Buszewicz, MRCGP, MRCPsych, Research Department of Primary Care \& Population Health, University College London, London; Scott Weich, MRCPsych, MD, Health Sciences Research Institute, University of Warwick, Warwick; Michael King, MRCPsych, PhD, Department of Mental Health Sciences, University College London, London, UK

Correspondence: Kate Walters, Research Department of Primary Care \& Population Health, Hampstead Campus, University College London, Rowland Hill St, London NW3 2PF, UK. Email: k.walters@ucl.ac.uk

First received 23 Jul 2010, final revision 10 Oct 2010, accepted 15 Dec 2010

\section{Funding}

This study was supported by a Medical Research Council Special Training Fellowship in Health Services Research (G106/1053) for the main author (K.W.).

\section{Acknowledgements}

We thank Susannah Payne for her help with collecting the data, and all study practices and participants.

\section{References}

1 Bijl RV, de Graaf R, Hiripi E, Kessler RC, Kohn R, Offord DR, et al. The prevalence of treated and untreated mental disorders in five countries. Health Aff (Milwood) 2003; 22: 122-33.

2 WHO World Mental Health Survey Consortium. Prevalence, severity and unmet need for treatment of mental disorders in the World Health Organization World Mental Health Surveys. JAMA 2004; 291: 2581-90.

3 Singleton N, Bumpstead R, O'Brien R, Lee A, Meltzer H. Psychiatric Morbidity among Adults Living in Private Households. TSO (The Stationery Office), 2001.

4 Frances A. The first draft of DSM-V. BMJ 2010; 340: C1168.

5 World Health Organization. The ICD-10 Classification of Mental and Behavioural Disorders: Clinical Descriptions and Diagnostic Guidelines. WHO, 1992.

6 American Psychiatric Association. Diagnostic and Statistical Manual of Mental Disorders (4th edn) (DSM-IV). APA, 1994.

7 American Psychiatric Association. Proposed Draft Revisions to DSM Disorders and Criteria. American Psychiatric Association, 2010 (http:// www.dsm5.org/ProposedRevisions/).

8 Barkow K, Heun R, Wittchen HU, Ustun TB, Gansicke M, Maier W. Mixed anxiety-depression in a 1 year follow-up study: shift to other diagnoses or remission? J Affect Disord 2004; 79: 235-9.

9 Cuijpers P, Smit F. Subthreshold depression as a risk indicator for major depressive disorder: a systematic review of prospective studies. Acta Psychiatr Scand 2004; 109: 325-31.

10 Forsell, Y. A three-year follow-up of major depression, dysthymia, minor depression and subsyndromal depression: results from a population-based study. Depress Anxiety 2007; 24: 62-5.

11 Hermens ML, van Hout HPJ, Terluin B, van der Windt D, Beekman A van Dyck $R$, et al. The prognosis of minor depression in the general population: a systematic review. Gen Hosp Psychiatry 2004; 26: 453-62.

12 Jackson JL, Passamonti $\mathrm{M}$, Kroenke $\mathrm{K}$. Outcome and impact of mental disorders in primary care at 5 years. Psychosom Med 2007; 69: 270-6.

13 Goldberg D. The General Health Questionnaire Users Manual. NFER Nelson, 1988. 
14 Walters K, Buszewicz M, Weich S, King M. Help-seeking preferences for psychological distress in primary care: effect of current mental state. Br J Gen Pract 2008; 58: 694-8.

15 Lewis G, Pelosi AJ, Glover E, Wilkinson G, Stansfeld SA, Williams P, et al. The development of a computerised assessment for minor psychiatric disorder. Psychol Med 1988; 18: 737-45.

16 Lewis G, Pelosi AJ, Araya R, Dunn G. Measuring psychiatric disorder in the community: a standardised assessment for use by lay interviewers. Psycho Med 1992; 22: 465-86.

17 Evans M, Kessler D, Lewis G, Peters TJ, Sharp D. Assessing mental health in primary care research using standardized scales: can it be carried out over the telephone? Psychol Med 2004; 34: 157-62.

18 Ormel J, Koeter MWJ, Van den Brink W, Giel R. Concurrent validity of GHQ-28 and PSE as measures of change. Psychol Med 1989; 19: 1007-13.

19 Goldberg D, Gater R, Sartorius N, Ustun TB, Piccineli M, Gureje O, et al. The validity of two versions of the GHO in the WHO study of mental illness in general health care. Psychol Med 1997; 27: 191-7.

20 Gandek B, Ware JE, Aaronson NK, Apolone G, Bjorner JB, Brazier JE, et al. Cross-validation of item selection and scoring for the SF-12 health survey in nine countries: results from the IQOLA project. J Clin Epidemiol 1998; 51: $1171-8$

21 Ware JE, Turner-Bowker D, Kosinski M, Gandek B. SF-12V2 How to Score Version 2 of the SF-12 Health Survey. QualityMetric Incorporated RI, 2005.

22 World Health Organization. Disability Assessment Schedule. World Health Organization, 2001 (http://www.who.int/icidh/whodas/index.html)

23 Kroenke K, Spitzer RL, Williams JB. The PHQ-15: validity of a new measure for evaluating the severity of somatic symptoms. Psychosom Med 2002; 64 258-66

24 King M, Nazareth I, Levy G, Walker C, Morris R, Weich S, et al. Prevalence of common mental disorders in general practice attendees across Europe. $\mathrm{Br} J$ Psychiatry 2008; 192: 362-7.

25 Ormel J, Oldehinkel T, Brilman E, Van den Brink W. Outcome of depression and anxiety in primary care: a three wave $3 \frac{1}{2}$ year study of psychopathology and disability. Arch Gen Psychiatry 1993; 50: 759-66.
26 Pini S, Perkonnig A, Tansella M, Wittchen HU, Psich D. Prevalence and 12 month outcome of threshold and subthreshold mental disorders in primary care. J Affect Disord 1999; 56: 37-48.

27 Das-Munshi J, Goldberg D, Bebbington PE, Bhugra DK, Brugha TS, Dewey ME, et al. Public health significance of mixed anxiety and depression: beyond current classification. Br J Psychiatry 2008; 192: 171-7.

28 Da Silva Lima A, de Almeida Fleck M. Subsyndromal depression: an impact on quality of life? J Affect Disord 2007; 100: 163-9

29 Simon G, Ormel J, von Korff M, Barlow W. Health care costs associated with depressive and anxiety disorders in primary care. Am J Psychiatry 1995; 152: 352-7.

30 Snyder S, Strain JJ, Wolf D. Differentiating major depression from adjustment disorder with depressed mood in the medical setting. Gen Hosp Psychiatry 1990; 12: 159-65.

31 Despland JN, Monod L, Ferrero F. Clinical relevance of adjustment disorder in DSM-III-R and DSM-IV. Compr Psychiatry 1995; 36: 454-60.

32 Tyrer $P$, Seivewright $H$, Johnson $T$. The Nottingham Study of Neurotic Disorder: predictors of 12-year outcome of dysthymic, panic and generalized anxiety disorder. Psychol Med 2004; 34: 1385-94.

33 Beard JR, Heathcote K, Brooks R, Earnest A, Kelly B. (2007) Predictors of mental disorders and their outcome in a community based cohort. SoC Psychiatry Psychiatr Epidemiol 2007; 42: 623-30.

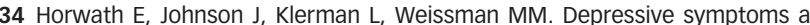
relative and attributable risk factors for first-onset major depression. Arch Gen Psychiatry 1992; 49: 817-23.

35 Kessler RC, Merikangas KR, Berglund P, Eaton WW, Koretz DS, Walters EE. Mild disorders should not be eliminated from the DSM-V. Arch Gen Psychiatry 2003; 60: 1117-22.

36 National Institute for Health and Clinical Excellence CG90 Depression in adults: NICE Guidance. NICE, 2009 (http://guidance.nice.org.uk/CG90/ NICEGuidance/). 